\title{
DETERMINANTES SOCIALES EN SALUD Y ESTILOS DE VIDA EN POBLACIÓN ADULTA DE CONCEPCIÓN, CHILE
}

\section{SOCIAL DETERMINANTS OF HEALTH AND LIFESTYLES IN ADULT POPULATION CONCEPCIÓN, CHILE}

\author{
DAISY VidAL GUTIÉRREZ \\ Isis Chamblas García ${ }^{* *}$ \\ Mercedes Zavala Gutiérrez ${ }^{* * *}$ \\ RalPh MÜller GILChrisT ${ }^{* * * *}$ \\ María Cecilia Rodríguez Torres ${ }^{* * * *}$ \\ Alejandra Chávez Montecino*****
}

\begin{abstract}
RESUMEN
Diversos son los factores que generan disparidades en el acceso al sistema de salud y en su utilización, lo que produce desigualdades tanto en la promoción de la salud como en las posibilidades del restablecimiento y supervivencia tras una enfermedad. El propósito del estudio fue determinar la relación entre estilo de vida promotor de salud y variables psicosociales, demográficas y de salud, en las comunas de Coronel y Concepción. Material y método: Corresponde a un estudio descriptivo y correlacional, de orientación cuantitativa. La población de estudio estuvo constituida por 441 adultos, de ambos sexos, entre 20 y 65 años, asistentes en el 2011 a establecimientos de atención primaria de salud. La muestra fue no aleatoria, con cuotas sexo y edad. El instrumento utilizado fue la Escala Health-Promoting Lifestyle Profile II. El procesamiento de la información se realizó con el Programa SAS. Resultados: Se encontró asociación significativa entre estilo de vida promotor en salud y determinantes estructurales como: el lugar de residencia, edad, sexo, educación, ingresos. Se encontraron asociaciones significativas en variables psicosociales como: percepción de problemas del entorno; satisfacción con aspectos de la vida; apoyo afectivo; apoyo y confianza; autoestima y autoeficacia. Conclusiones: Los resultados permiten reafirmar la relevancia de considerar, además de los factores estructurales, los factores psicosociales en la mirada y en la intervención en salud para potenciar estilos de vida promotores de salud. Los factores psicosociales se encuentran fuertemente asociados a estilos de vida promotores de salud.
\end{abstract}

Palabras clave: Determinantes sociales en salud, estilo de vida, promoción de la salud.

\footnotetext{
ABSTRACT

There are many factors that create disparities in access and use of health systems, resulting inequalities in both health promotion and the possibilities of recovery and survival after an illness. The purpose of the study was to determine the relationship between lifestyle health promoter and psychosocial, demographic and health

${ }^{*}$ Asistente Social, profesora Departamento de Trabajo Social, Universidad de Concepción, Concepción, Chile. Email: dvidal@udec.cl

${ }^{* *}$ Asistente Social, profesor Departamento de Trabajo Social, Universidad de Concepción, Concepción, Chile. Email: ichambla@udec.cl

${ }^{* * *}$ Enfermera, profesora Programa Magíster en Trabajo Social, Departamento de Trabajo Social, Concepción, Chile. Email: mzavala@udec.cl

${ }_{* * * *}^{*}$ Médico, Centro de Salud Familiar Dr. Víctor Manuel Fernández, Concepción, Chile. Email: rmuller@ssconcepcion.cl

${ }^{* * * *}$ Asistente Social, profesora Departamento de Trabajo Social, Universidad de Concepción, Concepción, Chile. Email: mrodriguezt@udec.cl

****** Asistente Social, Hospital de Coronel. Coronel, Chile. Email: achavez@ssconcepcion.cl
} 
variables, in the communes of Coronel and Concepción. Methods: It is a descriptive and correlational study, quantitative orientation. The study population consisted of 441 adults, both sexes, between 20 and 65 years old users of primary health care in 2011. The sample was not random, with quotas for gender and age. The instrument used to measure was the Scale Health-Promoting Lifestyle Profile II. The information processing was performed with SAS program. Results: A significant association was found between the life style health promoter and structural determinants such as place of residence, age, sex, education, income. Significant associations were found in psychosocial variables such as perception of environmental problems; satisfaction with aspects of life, emotional support, support and confidence, self-esteem and self-efficacy. Conclusions: Results suggest the need of considering, besides to structural factors, the psychosocial factors at the gaze and health intervention for enhancing health promoting life styles. Psychosocial factors are strongly associated with health promoting life styles.

Key words: Social determinants of health, life style, health promotion.

Fecha recepción: 24/01/13 Fecha aceptación: 27/12/13

\section{INTRODUCCIÓN}

Esta investigación se sitúa en el área de las Políticas Públicas en Chile, particularmente en el área de la Salud y en su componente de Promoción de la Salud y en relación con la adopción de estilos de vida saludable en la población chilena. Específicamente, interesa investigar acerca de la influencia de los Determinantes Sociales en Salud en los Estilos de vida de la población adulta de la comunas de Coronel y Concepción. La Encuesta Nacional de Salud 2009-2010 (1) evidencia para Chile, un complejo diagnóstico: las enfermedades crónicas no transmisibles representan el grueso de la carga de salud para el país, encontrándose éstas asociadas con hábitos que suelen adquirirse tempranamente en la infancia o en la adolescencia y que además se encuentran asociados a una compleja y múltiple red de determinantes. Los determinantes sociales de la salud que en su integralidad abordan elementos que intentan explicar que las desigualdades en que se manifiestan los resultados en salud para diversos grupos de la sociedad, dicen relación con las distintas posibilidades que tienen las personas de desarrollarse en la vida y gozar de buena salud. Esas desigualdades se observan en las condi- ciones de vida de la primera infancia, la escolarización, la naturaleza del empleo y las condiciones de trabajo, las características físicas del medio construido y la calidad del medio natural en que vive la población. Según el carácter de esos entornos, las condiciones físicas, el apoyo psicosocial y los esquemas de conducta varían para cada grupo, haciéndolos más o menos vulnerables a la enfermedad (2). La estratificación social también crea disparidades en el acceso al sistema de salud y en su utilización, lo que da lugar a desigualdades en la promoción de la salud y el bienestar, la prevención de enfermedades y las posibilidades de restablecimiento y supervivencia tras una enfermedad (3). A este respecto, el Ministerio de Salud de Chile ha emprendido un desafío significativo a través del compromiso con el diseño de una política pública en salud para la década 2011-2020, centrada en la generación de objetivos estratégicos que consideran específicamente los determinantes sociales como referentes relevantes para el entendimiento de la producción y abordaje de las inequidades en salud. Dado que las conductas de un ser humano son el resultado de la interacción entre variables individuales, sociales y ambientales, se ha tomado conciencia de la necesidad de modificar los enfoques en salud, siendo hoy el predomi- 
nante en salud el denominado "Enfoque de Determinantes Sociales de la Salud". Por otra parte, el concepto de la salud como derecho humano fundamental, consignado en la Carta de Ottawa (4), destaca determinados prerrequisitos para la salud, que incluyen la paz, adecuados recursos económicos y alimenticios, vivienda, un ecosistema estable y un uso sostenible de los recursos. El reconocimiento de estos prerrequisitos pone de manifiesto la estrecha relación que existe entre las condiciones sociales y económicas, el entorno físico, los estilos de vida individuales y la salud. Estos vínculos constituyen la clave para una comprensión holística de la salud que es primordial en la definición de la promoción de la salud. Una visión integral de la salud supone que todos los sistemas y estructuras que rigen las condiciones sociales y económicas, al igual que el entorno físico, deben tener en cuenta las implicaciones y el impacto de sus actividades en la salud y el bienestar individual y colectivo (5).

El concepto de determinantes sociales surge con fuerza desde hace dos décadas, ante el reconocimiento de las limitaciones de las intervenciones dirigidas a los riesgos individuales de enfermar, que no tomaban en cuenta el rol de la sociedad. Los argumentos convergen en la idea que las estructuras $\mathrm{y}$ patrones psicosociales forman $\mathrm{u}$ orientan las decisiones y oportunidades de ser saludables de los individuos. Así, una pregunta clave para las políticas de salud es: ¿hasta qué punto es la salud una responsabilidad social y no sólo una responsabilidad individual? Esta pregunta, planteada por la nueva Comisión sobre Determinantes Sociales de la Salud, es central al enfoque de determinantes sociales y amplía el foco de los esfuerzos en salud pública más allá de las personas, e incluso las comunidades, para analizar las causas sociales más estructurales (6). Como determinantes sociales de la salud se entienden las características específicas y las vías mediante las cuales las condiciones sociales afectan a la salud de las personas (7). Los determinantes so- ciales son tanto de carácter socio individual, tales como las prácticas de salud, capacidad y aptitudes para la adaptación de la persona, como aquéllos derivados de la estructura de una sociedad, entre los que se sitúan la pobreza, la educación, el trabajo, el género, el nivel socioeconómico. De esta manera, los determinantes sociales de la salud se refieren tanto al contexto social como a los procesos mediante los cuales las condiciones sociales se traducen en consecuencias para la salud" (8). El estudiar y conocer los determinantes sociales presentes como factores predictores de la adopción de estilos de vida promotores de salud, en una población determinada, permite generar insumos que pueden aportar elementos necesarios a considerar en la formulación de políticas públicas y programas, buscando el mayor impacto en cada una de ellas para la generación de conductas saludables en la población (9). Al respecto, la política de salud actual del país incorpora los conceptos de Promoción de la Salud y los Determinantes Sociales, encontrándose adscritos a las pautas generales de la Organización Mundial de la Salud (OMS), en especial a lo determinado por la Comisión de Determinantes Sociales. El último Congreso de Promoción de la Salud desarrollado en el país en enero de 2007 se denominó: "Determinantes Sociales en la Salud: Desafío para una mejor Calidad de Vida”. Después de esto, se formalizó, a través del Ministerio de Salud, el interés de colaborar como país socio de la Comisión y se elaboró un plan de trabajo con el objetivo de integrar el enfoque de determinantes sociales en la política de salud del Gobierno e intercambiar experiencias con otros países (6). Así, Chile asume el Modelo de Determinates en Salud que postula la Comisión del mismo nombre de la OMS en el año 2005. Este Modelo incorpora elementos relevantes a considerar como factores asociados y predictores en la adopción de estilos de vida promotores de salud: al contexto social, la estratificación social, la vulnerabilidad diferencial y los sistemas de salud. Desde esta 
mirada, los determinantes de la salud incluyen: el ambiente social y económico, el ambiente físico, y las características individuales y comportamientos de la persona. Ya en el año 1974, en el Informe de Lalonde (10) se identifican como determinantes de la salud: los estilos de vida, el ambiente, la biología humana y los servicios de salud, señalando que los esfuerzos por mejorar la salud de las personas no debieran concentrarse sólo en los servicios de salud, sino se requiere intervenir en los estilos de vida (11). Posterior a esto, Acheson, adaptando el modelo de Dahlgren y Whitehead, diagrama los determinantes de la salud como capas de influencia (12).

En dicho modelo, el centro está representado por la persona y sus características no modificables: edad, sexo, y que se proyectan hacia los estilos de vida del individuo, los que se encuentran determinados o influenciados por las redes sociales y comunitarias en las que se encuentran insertos el individuo y su grupo familiar, las cuales, a su vez, se encuentran condicionadas por otros factores predictores, tales como la educación, el ambiente laboral, las condiciones de vida y de trabajo, el empleo, el acceso a servicios básicos, la calidad de la vivienda y los servicios de atención de salud, todos éstos (10) influencian la adopción de estilos de vida saludable.

El campo de los determinantes sociales de la salud es quizás uno de los más complejos y desafiantes. Se trata de los aspectos claves de la vida y las circunstancias laborales de las personas y de sus estilos de vida que explican en gran proporción la diferencia en muchos de los indicadores de salud. Wilkinson y Pickett (13) plantean que las diferencias en salud son no sólo una gran injusticia social, sino que también atraen la atención de los científicos sobre algunos de los más impactantes determinantes de los niveles de salud de las sociedades modernas. Más concretamente, han permitido una mayor comprensión de la extraordinaria susceptibilidad de la salud ante el entorno social. Temas como la pobreza, las drogas, las condiciones laborales, el desempleo, el apoyo social, la buena alimentación y la política de transporte, son algunos de los determinantes que actúan fuertemente sobre las personas desde la primera infancia (13). Otros elementos que inciden poderosamente en la salud y en los estilos de vida promotores de salud son las variables sociológicas y psicológicas: el comportamiento es un eslabón ineludible en la cadena causal de la mayoría de los determinantes ambientales y genéticos y los consecuentes efectos que tienen para la salud. Por su parte, el entorno social presenta una complejidad mayor como mediador y moderador entre la conducta y el medio ambiente en la determinación de la salud de la población (13).

Si se combina la economía, la sociología y la psicología con la medicina y la neurobiología, parece como si todo dependiera en gran medida de la comprensión de la interacción entre las desventajas materiales y sus implicaciones sociales; no es simplemente que las circunstancias de pobreza material sean dañinas para la salud; el significado social de la pobreza, el desempleo, la marginación social, o de otros estigmas son relevantes (14). La inequidad adquiere aún mayor relevancia al considerar que las circunstancias económicas y sociales asociadas a la pobreza afectan a la salud de por vida. Las personas que viven en los peldaños más bajos de la escala social suelen estar sometidas a un riesgo doble de padecer enfermedades graves y muertes prematuras que las personas que viven más cerca de los peldaños superiores (3). Así también, el ser humano, en calidad de ser social, no necesita solamente contar con unas buenas condiciones materiales, sino que ya desde la primera infancia requiere sentirse valorado y apreciado; necesita soporte social, de sociedades más amigables, necesita sentirse útil y tener un cierto nivel de control sobre un trabajo valioso. Sin ello, la persona se torna más propensa a la depresión, al uso de las drogas, a la ansiedad, la hostilidad y a la desesperación, lo que repercute en la salud física (3). Un estado de ansiedad prolongado, la inse- 
guridad, una autoestima baja, el aislamiento social y la falta de control sobre el trabajo y la vida doméstica ejercen unos efectos impactantes sobre la salud. Esos tipos de riesgos psicosociales se van acumulando a lo largo de toda la vida y aumentan las posibilidades de padecer una alteracón de salud mental y una muerte prematura (15). Ser excluido de la vida social y recibir un tratamiento de inferioridad causa la peor salud y los riesgos más elevados de padecer una muerte prematura y más concretamente, el estrés que produce vivir en la pobreza es particularmente perjudicial durante el embarazo para los bebés, los niños y los ancianos (3). Como otro elemento muy relevante se debe mencionar una buena dieta y un suministro de comida adecuado, los que constituyen dos de los factores más determinantes en la promoción de la salud y el bienestar. La carencia de comida o la falta de variedad provocan desnutrición y enfermedades carenciales, a la vez, un consumo excesivo contribuye al desarrollo de enfermedades cardiovasculares, diabetes, cáncer, enfermedades oculares degenerativas, obesidad y caries dentales (16). En esta lógica, las condiciones sociales y económicas se traducen en un gradiente social en la calidad de la dieta que contribuye a acentuar la desigualdad en salud. La posición o nivel socioeconómico es una variable ampliamente utilizada en investigación en salud, es un concepto multidimensional y no existe un único estándar para su medición. Se reconocen para este concepto tres indicadores clásicos: ingreso, educación y ocupación (17, 18). Las diferencias socioeconómicas en salud se reflejan en las medidas generales de salud, como la esperanza de vida, la mortalidad por todas las causas, y autopercepción de salud. La situación socioeconómica también está relacionada con la prevalencia y el curso de la enfermedad y la autovaloración de la salud. Las desigualdades socioeconómicas en salud son evidentes en las causas específicas de la enfermedad, discapacidad y muerte prematura, incluyendo cáncer de pulmón, enfermedad coronaria, accidentes y suicidio (19). Según lo reporta la literatura internacional existen grandes diferencias en las tasas de morbimortalidad en los distintos grupos sociales, tanto en Chile como en el resto del mundo (20). En Chile, entre los años 1998 y 2006, la esperanza de vida a los 20 años aumentó en 1,5 años, pero este incremento no fue homogéneo segun nivel educacional, para el grupo de varones sin escolaridad el aumento fue de 0,8 años, mientras que para el grupo con 13 y más años de educación el aumento fue de 2,8 años (21). La importancia de la posición social también se observa en diversos factores de riesgo para la salud: la obesidad, exceso de peso y sedentarismo es mayor en la población con menor escolaridad (21). También es posible observar la relación entre posición social y salud desde una perspectiva de ciclo vital: la población adulta chilena en desventaja tiene mayor tendencia a enfermar y sus hijos mantienen esa condición hasta por 20 años más, la probabilidad de enfermar de cualquier chileno en la adultez está, al menos parcialmente, determinada por la posición social de su familia de origen (22). La perspectiva de curso de vida, indica que la prevención de factores de riesgo y problemas de salud en la adultez exige modificar sus desencadenantes desde la vida temprana (23).

Todos estos factores, y sin duda otros más, contribuyen a generar grandes diferencias en las condiciones de salud que repercuten en diversos resultados en salud.

El objetivo de este trabajo es determinar la relación entre estilo de de vida promotor de salud y variables psicosociales, demográficas y de salud.

\section{MATERIAL Y MÉTODO}

Corresponde a una investigación cuantitativa, descriptiva y correlacional. El universo estuvo conformado por población adulta entre 
20-64 años, que durante el año 2011 concurre a consultas de salud en tres centros públicos de salud de las comunas de Coronel y Concepción. Se trabajó con una muestra intencionada, que fue seleccionada a partir de los sujetos que se encontraban en el momento de aplicación de los instrumentos en el centro de atención de salud. Se trabajó con una muestra por cuotas diferenciada por sexo y edad para cada establecimiento prestador de la atención de salud. Se obtuvo una muestra final de 441 personas. Se excluyeron de la muestra sujetos con algún tipo de discapaciad mental, motora, visual o auditiva. Las entrevistas fueron realizadas por trabajadoras sociales y estudiantes de $5^{\circ}$ año de Trabajo Social, su aplicación fue realizada en las salas de espera de los centros de salud, se solicitó la firma del consentimiento informado a cada persona que reunía los requisitos para esta investigación. La variable dependiente es el estilo de vida promotor de salud. Las variables independientes o predictoras son edad, sexo, estado de salud, sistema de cuidados de salud, factores ambientales y disponibilidad y uso de las redes sociales. La medición del estilo de vida promotor de la salud se efectuó con la escala Health-tilo Promoting Lifestyle Profile II, versión en español $(24,25)$. Este instrumento está basado en el Modelo de Nola Pender, ha sido validado a nivel internacional, obteniendo como resultados que los seis factores explican el 45,9\% de la varianza. En el análisis factorial de segundo orden dio como resultado la obtención de un solo factor, interpretado como el estilo de vida promotor de salud. El coeficiente de confiabilidad alfa para la escala total fue de 0,93 , la fiabilidad test-retest a las dos semanas fue de 0,86 ; coeficientes alfa de las subescalas oscilaron desde 0,70 hasta 0,87 (24). En el instrumento indicado, el estilo de vida promotor de salud se concibe como un constructo multidimensional que incluye 6 dimensiones: Relaciones interpersonales; Nutrición; Responsabilidad en salud; Actividad física; Manejo del estrés; Crecimiento espiritual. Cada indicador se califica con una puntuación de 1 a 4, donde 1 es nunca y 4 es siempre, asumiendo que un mayor puntaje representa un estilo de vida promotor de salud más saludable. Para precisar las variables psicosociales, demográficas y de salud se construyó un instrumento ad hoc en base al Modelo de Determinantes Sociales de la OMS. El análisis de la información se realizó en base al Programa estadístico SAS, versión 9.1. Para el análisis descriptivo y correlacional de variables se utilizó t de Student y ANOVA. Se efectuó análisis de las características sicométricas de la variable dependiente, obteniéndose un alfa Cronbach de 0.923, congruente con los resultados obtenidos en el estudio de validación original del instrumento.

\section{RESULTADOS}

La población de estudio fue mayoritariamente del sexo femenino $(68,8 \%)$, relación de 3:1 mujeres v/s hombres, esto responde a la asignación por cuota realizada en la conformación de la población de estudio asumiendo antecedentes empíricos del acceso que realizan las mujeres a los centros de salud en nuestro país, que es en la relación 3:1 respecto del género masculino. Respecto de la edad, la categoría que acumula la mayor proporción corresponde a "joven", con un $34,7 \%$, distribuyéndose el porcentaje restante en las otras categorías en forma equitativa. En relación al Sistema Previsional se destaca para esta población que sólo el 30,6\% es cotizante activo en Administradoras de Fondos de Pensiones (AFP), un $12,7 \%$ se mantiene aún en el Instituto de Previsión Social (IPS), y un alto porcentaje $18,8 \%$ no se encuentra cotizando actualmente, un 27,9\% tampoco cotiza y corresponde mayoritariamente a mujeres dueñas de casa. En el nivel de escolaridad destaca que los mayores porcentajes corresponden a educación media, con un $53,9 \%$, la educación técnica y universitaria alcanza en total un 31,5\% (Tabla 1). 
Tabla 1. Descripción sociodemográfica según comunas.

\begin{tabular}{|c|c|c|c|c|c|c|}
\hline \multirow[b]{3}{*}{ Sexo } & \multicolumn{4}{|c|}{ Comuna } & & \\
\hline & \multicolumn{2}{|c|}{ Concepción } & \multicolumn{2}{|c|}{ Coronel } & \multicolumn{2}{|c|}{ Total } \\
\hline & $\mathbf{N}$ & $\%$ & $\mathbf{N}$ & $\%$ & $\mathbf{N}$ & $\%$ \\
\hline Masculino & 30 & 6,8 & 109 & 24,7 & 139 & 31,5 \\
\hline Femenino & 190 & 43,1 & 112 & 25,4 & 302 & 68,5 \\
\hline Total & 220 & & 221 & & 441 & 100,0 \\
\hline \multicolumn{7}{|l|}{ Edad } \\
\hline Joven & 82 & 18,6 & 71 & 16,1 & 153 & 34,7 \\
\hline Adulto Joven & 40 & 9,1 & 56 & 12,7 & 96 & 21,8 \\
\hline Adulto Medio & 47 & 10,7 & 46 & 10,4 & 93 & 21,1 \\
\hline Adulto Mayor & 51 & 11,6 & 48 & 10,9 & 99 & 22,5 \\
\hline Total & 220 & 49,9 & 221 & 50,1 & 441 & 100,0 \\
\hline \multicolumn{7}{|l|}{ Educación (Último año aprobado) } \\
\hline Básica & 42 & 9,5 & 22 & 4,9 & 64 & 14,5 \\
\hline Media & 117 & 26,5 & 121 & 27,4 & 238 & 54,0 \\
\hline Técnico Prof. y Univers. & 61 & 13,8 & 78 & 17,7 & 139 & 31,5 \\
\hline Total & 220 & 50,0 & 221 & 50,0 & 441 & 100,0 \\
\hline \multicolumn{7}{|l|}{ Previsión } \\
\hline No corresponde & 48 & 10,9 & 79 & 17,9 & 127 & 28,8 \\
\hline Cotizac. obligat. en AFP & 57 & 12,9 & 78 & 17,7 & 135 & 30,6 \\
\hline Cotizac. Obligat. IPS & 2 & 0,5 & 54 & 12,2 & 56 & 12,7 \\
\hline Capredena & - & - & 8 & 1,8 & 8 & 1,8 \\
\hline Dipreca & - & - & 1 & 0,2 & 1 & 0,2 \\
\hline Imponente Voluntario AFP & 1 & 0,2 & - & - & 1 & 0,2 \\
\hline Cotiza, no sabe donde & 1 & 0,2 & - & - & 1 & 0,2 \\
\hline No está cotizando actualmente & 82 & 18,6 & 1 & 0,2 & 83 & 18,8 \\
\hline No sabe / No contesta & 29 & 6,6 & _ & _- & 29 & 6,6 \\
\hline Totales & 220 & 49,9 & 221 & 50,1 & 441 & 100,0 \\
\hline \multicolumn{7}{|l|}{ Ingresos Grupo Familiar } \\
\hline (\$100.000 a \$199.999) & 72 & 16,3 & 34 & 7,7 & 106 & 24,0 \\
\hline Medio Bajo (\$300.000 a \$399.000) & 98 & 22,2 & 130 & 29,5 & 228 & 51,7 \\
\hline Medio Alto $\quad(\$ 600.000$ a \$799.000) & 40 & 9,1 & 53 & 12,0 & 93 & 21,1 \\
\hline (\$1.000.000 y más) & 10 & 2,3 & 4 & 0,9 & 14 & 3,2 \\
\hline Totales & 220 & 49,9 & 221 & 50,1 & 441 & 100,0 \\
\hline
\end{tabular}

Al explorar las diferencias en Estilos de vida promotor de salud de la población estudiada según comuna de residencia, se encontraron diferencias significativas a nivel de Estilo de vida promotor de salud en su medición global $(p=0.0274)$ y en las subescalas Relaciones Interpersonales $(\mathrm{p}=0.0007)$, Nutrición $(\mathrm{p}=0.020)$, Manejo del Estrés $(\mathrm{p}=0.0442)$ y Crecimiento espiritual $(\mathrm{p}<0.0001)$; en todas ellas es el grupo procedente de la comuna de Coronel quien presenta promedios más altos que los del sector de Concepción, incluso a nivel de las escalas donde no hay diferencias significativas (Tabla 2). 
Tabla 2. Estilos de Vida Promotor de Salud según comuna de residencia.

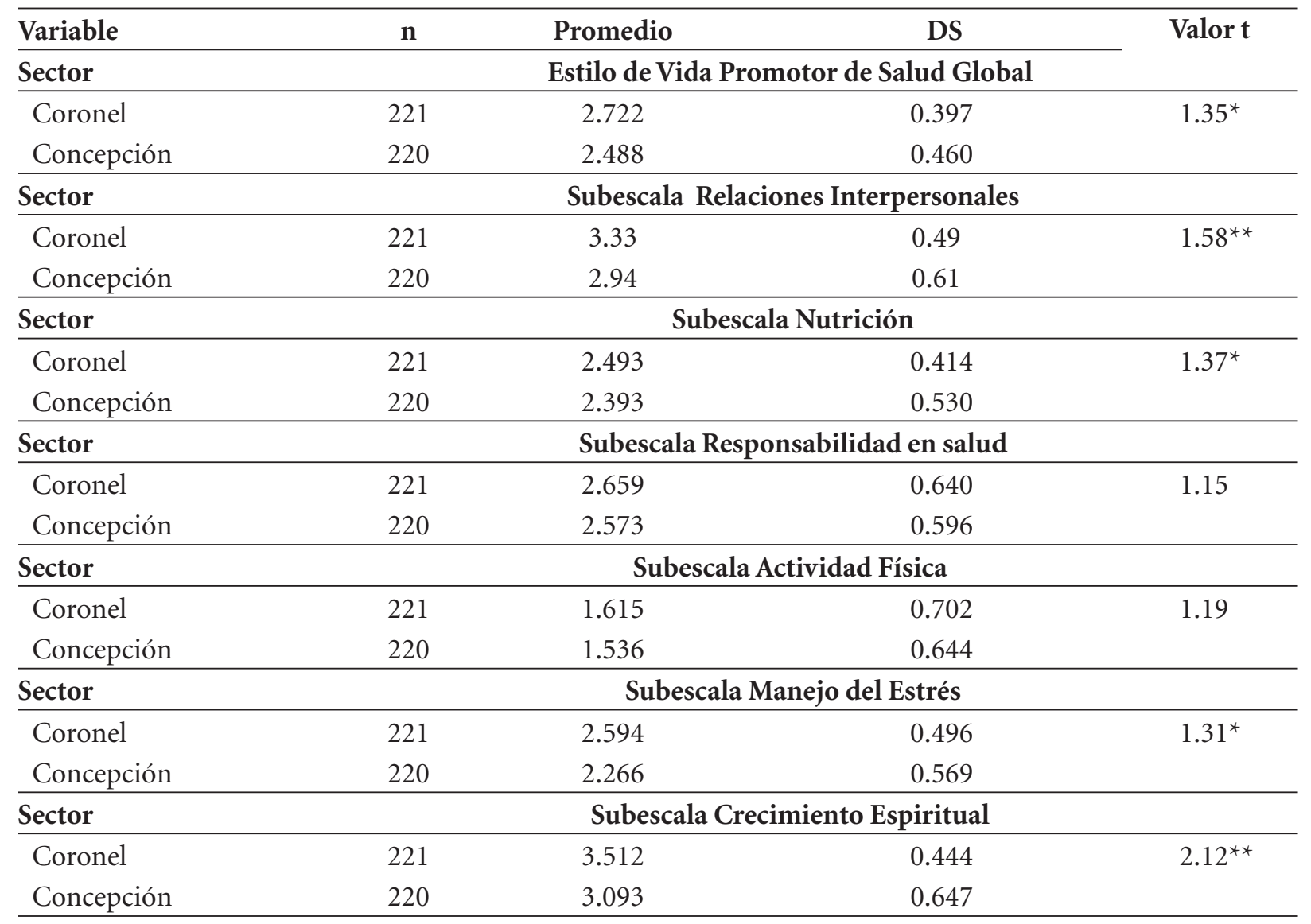

${ }^{*} \mathrm{p}<0.05,{ }^{* *} \mathrm{p}<0.01$

Al explorar diferencias por sexo, destacan como relevantes las diferencias significativas encontradas entre hombres y mujeres en las escalas: actividad física, manejo de estrés y crecimiento espiritual (Tabla 3 ).

Tabla 3. Estilo de Vida Promotor de Salud global y por subescalas según sexo (N=441).

\begin{tabular}{|c|c|c|c|c|c|}
\hline \multirow[b]{3}{*}{ Variable Dependiente: Estilo de Vida promotor de salud } & \multicolumn{4}{|c|}{ Sexo } & \multirow{3}{*}{$\begin{array}{c}\text { Valor } \\
\text { Prueba } \\
\mathbf{t}\end{array}$} \\
\hline & \multicolumn{2}{|c|}{ Hombre } & \multicolumn{2}{|c|}{ Mujer } & \\
\hline & Media & DS & Media & DS & \\
\hline & $\mathrm{n}=139$ & $\mathrm{n}=139$ & $\mathrm{n}=302$ & $\mathrm{n}=302$ & \\
\hline Escala Global & 2.681 & 0.402 & 2.571 & 0.460 & 1.31 \\
\hline Subescala Relaciones Interpersonales & 3.205 & 0.544 & 3.098 & 0.605 & 1.24 \\
\hline Subescala Nutrición & 2.435 & 0.452 & 2.45 & 0.514 & 1.30 \\
\hline Subescala Responsabilidad en salud & 2.646 & 0.629 & 2.551 & 0.594 & 1.12 \\
\hline Subescala Actividad Física & 2.551 & 0.733 & 2.646 & 0.634 & $1.34^{*}$ \\
\hline Subescala Manejo del Estrés & 2.576 & 0.466 & 2.363 & 0.584 & $1.57^{\star *}$ \\
\hline Subescala Crecimiento Espiritual & 3.472 & 0.492 & 3.226 & 0.619 & $1.58^{\star *}$ \\
\hline
\end{tabular}


Al relacionar el estilo de vida promotor de salud con la edad de las personas encuestadas, se encontraron diferencias significativas a nivel de las subescalas: relaciones interpersonales $(\mathrm{F}=3,93, \mathrm{p}=0.0087)$, responsabilidad en Salud $(\mathrm{F}=3,92, \mathrm{p}<.01)$, actividad física $(\mathrm{F}=6,9, \mathrm{p}=0.0002)$ y crecimiento espiritual $(\mathrm{F}=2,72 \mathrm{p}=0.0443)$. En el caso de la subescala relaciones interpersonales y crecimiento espiritual, las diferencias se presentan entre el grupo etario de 31 a 40 años respecto del grupo de 51 a 60 años, donde los primeros presentan promedios más altos y los segundos los promedios más bajos. Respecto de la responsabilidad en salud, el grupo más joven es el que tiene los promedios más bajos, difiere significativamente de todos los restantes grupos etarios, los que a su vez no difieren entre ellos respecto de estas conductas. Para la subescala actividad física se presentan tres grupos diferenciados significativamente entre sí. El grupo más joven (20 a 30 años, $\mathrm{X}=1.73$ ) son los que presentan los promedios más altos y son quienes difieren respecto de los adultos (mayores de 40 a 60 años). A su vez, el grupo entre 31 y 50 años difiere también respecto del grupo de 50 años y más, pero no de los más jóvenes que ellos. Los promedios a nivel de esta subescala indican que los jóvenes realizan más actividad física, sin embargo tampoco es algo frecuente, sino "algunas veces" ( $\mathrm{X}=1.73)$ y decrece a medida que aumenta la edad, por tanto los mayores de 50 a 60 años tienen el promedio más bajo con solo 1.36 puntos (en escala de 1 a 4), es decir, mayoritariamente "nunca" o "casi nunca" realizan ejercicio físico.

Cuando se relaciona el estilo de vida promotor de salud con educación, sólo se encuentran diferencias estadísticamente significativas a nivel de las subescalas: relaciones interpersonales $(\mathrm{p}=0.0332)$ y actividad física $(\mathrm{p}=0.0039)$, en ambas subescalas, quienes tienen educación superior presentan promedios más altos que quienes tienen educación media o básica.

En relación a la variable ingresos econó- micos, a nivel de Estilo de vida promotor de salud global, los promedios decrecen según decrece el nivel de ingresos, sin embargo las diferencias significativas se presentan sólo entre los grupos extremos $(\mathrm{p}<0.0001)$. En la subescala relaciones interpersonales las diferencias significativas se presentan entre los grupos de mayores ingresos respecto del de menores ingresos $(\mathrm{p}<0.0001)$. A nivel de la subescala nutrición, es el grupo de ingreso de 400 a 800 mil quien difiere con los de ingresos más bajos (menos de 200 mil), quienes cuentan con más de 800 mil pesos al mes, son similares a quienes cuentan con 200 a 400 mil, y no difieren significativamente con los restante grupos económicos $(\mathrm{p}=0.0005)$. En actividad física hay tres grupos que difieren entre sí, el promedio es más alto en quienes tienen ingresos superior a 800 mil pesos, que difieren con el grupo que recibe entre $400 \mathrm{y}$ 800 mil pesos y luego en una sola categoría y con los promedios más bajos quienes reciben 400 mil pesos y menos al mes $(\mathrm{p}<0.0001)$; En la subescala manejo del estrés, los dos grupos de ingresos superiores tienen promedios más altos y difieren significativamente del grupo de menor ingreso $(p=0.0011)$. Finalmente, en la escala crecimiento espiritual, quienes reciben entre 200 mil y 799 mil pesos se diferencian significativamente de quienes tienen los ingresos más bajos (Tabla 4).

Respecto de la relación entre Estilo de vida promotor de salud y variables psicosociales, se observó asociación significativa a nivel del Estilo de vida promotor de salud global y de cada una de las seis subescalas del instrumento con cada uno de los seis componentes psicosociales indagados: Percepción de problemas del entorno; Satisfacción con aspectos de la vida; Apoyo afectivo; Apoyo y confianza; Autoestima; Autoeficacia. En lo específico, respecto a autoestima, autoeficacia y satisfacción con aspectos de la vida que experimenta la persona, cada una de estas variables se correlaciona de manera directa y significativa con el estilo de vida promotor de salud, tanto en su medición global como 
Tabla 4. Estilo de Vida Promotor de Salud global y por subescalas según variables sociodemográficas $(\mathrm{N}=441)$.

\begin{tabular}{|c|c|c|c|c|c|c|c|}
\hline \multirow[b]{2}{*}{$\begin{array}{c}\text { Variable } \\
\text { Independiente }\end{array}$} & \multicolumn{7}{|c|}{ Puntaje Promedio Estilo de Vida promotor de salud } \\
\hline & $\begin{array}{l}\text { Escala } \\
\text { Global }\end{array}$ & $\begin{array}{c}\text { Subescala } \\
\text { Relaciones } \\
\text { Interpersonales }\end{array}$ & $\begin{array}{l}\text { Subescala } \\
\text { Nutrición }\end{array}$ & $\begin{array}{c}\text { Subescala } \\
\text { Responsabilidad } \\
\text { en salud }\end{array}$ & $\begin{array}{c}\text { Subescala } \\
\text { Actividad } \\
\text { Física } \\
\end{array}$ & $\begin{array}{l}\text { Subescala } \\
\text { Manejo } \\
\text { del Estrés } \\
\end{array}$ & $\begin{array}{c}\text { Subescala } \\
\text { Crecimiento } \\
\text { Espiritual }\end{array}$ \\
\hline \multicolumn{8}{|l|}{ Edad } \\
\hline 20-30 años & 2.595 & 3.123 & 2.381 & 2.479 & 1.729 & 2.416 & 3.327 \\
\hline $31-40$ años & 2.700 & 3.287 & 2.489 & 2.703 & 1.632 & 2.545 & 3.411 \\
\hline $41-50$ años & 2.608 & 3.124 & 2.500 & 2.694 & 1.491 & 2.405 & 3.287 \\
\hline $51-60$ años & 2.527 & 3.002 & 2.440 & 2.669 & 1.364 & 2.365 & 3.176 \\
\hline Valor Prueba F & 2.54 & $3.93^{* *}$ & 1.50 & $3.92^{* *}$ & $6.9^{*}$ & 1,93 & $2.72^{*}$ \\
\hline \multicolumn{8}{|l|}{ Ingresos (miles) } \\
\hline 800 y más & 2.831 & 3.246 & 2.429 & 2.818 & 2.491 & 2.625 & 3.318 \\
\hline $200-399$ & 2.595 & 3.154 & 2.425 & 2.618 & 1.808 & 2.585 & 3.437 \\
\hline $400-799$ & 2.761 & 3.342 & 2.628 & 2.650 & 1.450 & 2.422 & 3.353 \\
\hline 200 y menos & 2.463 & 2.886 & 2.331 & 2.534 & 1.522 & 2.287 & 3.077 \\
\hline Valor Prueba F & $9.11^{* *}$ & $11.13^{* *}$ & $5.97^{\star *}$ & 0,95 & $16.79^{* *}$ & $5.47^{* *}$ & $7.63^{\star *}$ \\
\hline \multicolumn{8}{|l|}{ Educación } \\
\hline Básica & 2.614 & 3.094 & 2.475 & 2.627 & 1.554 & 2.463 & 2.298 \\
\hline Media & 2.572 & 3.092 & 2.407 & 2.563 & 1.455 & 2.398 & 3.288 \\
\hline Superior & 2.678 & 3.269 & 2.496 & 2.724 & 1.762 & 2.472 & 3.346 \\
\hline Valor Prueba F & 1.98 & $3.43^{*}$ & 1.46 & 2.53 & $5.62^{*}$ & 0.86 & 0.33 \\
\hline
\end{tabular}

${ }^{*} \mathrm{p}<0.05,{ }^{* *} \mathrm{p}<0.01$

Tabla 5. Correlación $\mathrm{r}$ de Pearson Estilos de Vida promotor de salud, global y subescalas según variables psicosociales.

\begin{tabular}{|c|c|c|c|c|c|c|}
\hline ESTILOS DE VIDA & $\begin{array}{c}\text { Percepción } \\
\text { Problemas } \\
\text { del entorno } \\
(\mathrm{n}=441)\end{array}$ & $\begin{array}{c}\text { Satisfacción } \\
\text { Aspectos } \\
\text { de la vida } \\
(\mathbf{n}=440) \\
\end{array}$ & $\begin{array}{l}\text { Apoyo } \\
\text { Afectivo } \\
(\mathrm{n}=441)\end{array}$ & $\begin{array}{c}\text { Apoyo y } \\
\text { Confianza } \\
(n=441)\end{array}$ & $\begin{array}{c}\text { Autoestima } \\
(\mathrm{n}=441)\end{array}$ & $\begin{array}{c}\text { Autoeficacia } \\
(\mathbf{n}=220)\end{array}$ \\
\hline Estilo de Vida Global $(\mathrm{N}=281)$ & $0.17^{\star *}$ & $0.38^{\star *}$ & $0.27^{\star *}$ & $0.30^{* *}$ & $0.36^{\star *}$ & $0.61^{* *}$ \\
\hline Relaciones Interpersonales & $0.18^{\star *}$ & $0.40^{* *}$ & $0.35^{\star *}$ & $0.35^{\star *}$ & $0.33^{\star *}$ & $0.57^{\star \star}$ \\
\hline Nutrición & 0.07 & $0.19^{* *}$ & $0.18^{\star *}$ & $0.19^{* *}$ & $0.19^{\star *}$ & $0.39^{* *}$ \\
\hline Responsabilidad en Salud & 0.01 & $0.19^{* *}$ & $0.23^{* *}$ & $0.21^{\star *}$ & $0.27^{\star *}$ & $0.51^{\star *}$ \\
\hline Actividad Física & 0.07 & $0.18^{\star *}$ & 0.02 & 0.07 & $0.22^{\star *}$ & $0.24^{\star *}$ \\
\hline Manejo del Estrés & $0.23^{\star *}$ & $0.38^{\star *}$ & $0.21^{\star *}$ & $0.23^{\star *}$ & $0.24^{\star \star}$ & $0.40^{\star *}$ \\
\hline Crecimiento Espiritual & $0.21^{\star *}$ & $0.39^{\star *}$ & $0.28^{\star *}$ & $0.30^{* *}$ & $0.37^{\star *}$ & $0.65^{\star *}$ \\
\hline
\end{tabular}

${ }^{\star *} \mathrm{p}<0.01$

en cada una de las subescalas (significación superior al 1 por 1.000).

En otro ámbito, aunque ciertamente rela- (ambas asociadas a la percepción de apoyo 
que experimenta la persona) se encontraron correlaciones significativas y directas a nivel de Estilo de vida promotor de salud global, como por cada una de las subescalas, a excepción de la subescala actividad física. En lo que respecta a la variable Percepción de problemas del entorno, se encontró asociación significativa inversa con el Estilo de vida promotor de salud global y con las subescalas: Relaciones interpersonales; Manejo del estrés y Crecimiento espiritual. Es decir, quienes perciben una menor cantidad de problemas en el entorno, son quienes evidencian mayores puntajes en Estilo de vida promotor de salud a nivel global y en cada una de las subescalas indicadas. Para las subescalas: $\mathrm{Nu}-$ trición, Responsabilidad en salud y Actividad física, quienes perciben una mayor cantidad de problemas en el entorno son aquellos que evidencian menores puntajes en Estilo de vida promotor de salud (Tabla 5).

\section{DISCUSIÓN Y CONCLUSIÓN}

Al explorar diferencias en los estilos de vida promotores de salud de acuerdo a los distintos predictores indagados se encuentran diferencias estadísticamente significativas en Estilo de Vida promotor de salud respecto del lugar de residencia de la población estudiada, tanto en su medición global como en las subescalas de Relaciones interpersonales; Nutrición; Manejo del estrés y Crecimiento espiritual; en todas ellas es el grupo procedente de la comuna de Coronel quien presenta promedios más altos que los del sector de Concepción. Esta situación llama la atención, dado que Coronel es un sector de mayor vulnerabilidad social que Concepción y por ello se esperaban promedios si bien no bajos, no superiores a Concepción. Al respecto, un elemento relevante a considerar a futuro debieran ser las características de la red de atención de salud para cada población estudiada, asumiendo que la red de establecimientos de salud se constituye en un relevante determinante de la salud, tanto en la perspectiva de las posibilidades de acceso como tipo y calidad de prestaciones. Relevante es también señalar que si bien la comuna de Coronel reúne mayores condiciones de vulnerabilidad social que Concepción, es una población que se encuentra muy integrada a la comuna y redes institucionales de Concepción.

Respecto de la variable sexo se encuentran diferencias significativas en tres de las 6 subescalas que componen este comportamiento global: Actividad física ( $\mathrm{t}=1.34, \mathrm{p}=0.0402)$, Manejo del estrés $(t=1.57, \mathrm{p}=0.0029)$ y Crecimiento espiritual $(\mathrm{t}=1.58, \mathrm{p}=0.0024)$. En todos los casos mencionados, los hombres son quienes tienen promedios más altos respecto de las mujeres, esto no es congruente con lo encontrado por otros autores, como Cid et al.(26) en Estilo de Vida Promotor de Salud (EVPS) Global, y Chávez (27). En relación con la edad se encontraron diferencias significativas a nivel de las subescalas: Relaciones interpersonales, Responsabilidad en salud, Actividad física y Crecimiento espiritual. Son las personas de edad media (31 a 40 años) quienes presentan los promedios más altos en Estilo de vida promotor de salud. Lo expuesto no concuerda con otros trabajos donde se aprecia una tendencia directa entre edad y conductas promotoras de salud $(5,26)$. Cuando se relaciona el estilo de vida promotor de salud con la Educación de las personas, agrupadas en las categorías básico, medio y superior, los resultados no muestran con claridad la tendencia esperada que indica que quienes tienen mayor educación, presentan promedios más altos en estos comportamientos. En la muestra estudiada, dichas diferencias fueron significativas sólo a nivel de las subescalas Relaciones interpersonales y en Actividad física. Sólo para estas escalas se evidenció que quienes tienen educación superior presentan promedios más altos en el Estilo de vida promotor de salud 
que quienes tienen educación media y/o básica. La variable ingresos está directamente relacionada con los estilos de vida, esto se presenta con diferencias significativas en 5 de las 6 subescalas, además de la comparación a nivel global (solo la medición en subescala Responsabilidad en salud no evidencia estas diferencias). El Nivel Socioeconómico es una variable relevante de consignar en la medida que es una variable que está asociada a las situaciones que permiten a las personas tener capacidades y recursos para lograr llevar a la práctica la predisposición existente de determinadas conductas, positivas o negativas (28).

Finalmente es relevante destacar que se encontraron asociaciones significativas entre el estilo de vida promotor de salud en su medición global, con cada una de las variables psicosociales indagadas: percepción de problemas del entorno; satisfacción respecto de distintos aspectos de la vida; apoyo afectivo; apoyo y confianza; autoestima y autoeficacia. Estos dos últimos elementos han sido encontrados fuertemente relacionados y como predictores de estilos de vida promotor de salud por Cid et al. (26), Triviño et al. (5) y Gamarra et al. (29). Lo anterior reafirma la necesidad de abordar la promoción de la salud desde una mirada multidimensional. La generación de estilos promotores de salud y por consiguiente la disminución de alteraciones de la salud están fuertemente asociadas a elementos sociales, culturales y psicosociales.

En conformidad a los hallazgos encontrados se puede reafirmar que la investigación en el campo de los determinantes sociales en salud adquiere gran relevancia, dado a que tradicionalmente en las políticas de salud han predominado las soluciones centradas en el tratamiento de las enfermedades, sin incorporar adecuadamente intervenciones sobre las "causas de las causas", tales como, las acciones sobre el entorno psicosocial (3).

\section{REFERENCIAS}

1. Ministerio de Salud (MINSAL), Gobierno de Chile. Encuesta Nacional de Salud 2009-2010 [Internet]. Santiago, Chile: MINSAL; 2009 [citado el 11 de noviembre de 2013]. Disponible en: http://epi. minsal.cl

2. Organización Mundial de la Salud (OMS) Comisión sobre Determinantes Sociales de la Salud. Subsanar las desigualdades en una generación. Alcanzar la equidad sanitaria actuando sobre los determinantes sociales de la salud. Buenos Aires, Argentina: OMS; 2009.

3. Comisión sobre Determinantes Sociales de la Salud. Acción sobre los factores sociales determinantes de la salud: Aprender de las experiencias anteriores. Ginebra: Comisión sobre Determinantes Sociales de la Salud. 2005.

4. Unidad de promoción de la Salud y Educación para la Salud, de la División de Comunicación, Educación y Promoción de la Salud de la Organización Mundial de la Salud. Promoción de la Salud, glosario. Ginebra: OMS; 1998.

5. Triviño Z, Stiepovich J, Merino J. Factores predictores de conductas promotoras de salud en mujeres peri-post-menopáusicas de Cali, Colombia. Colom Med 2007; 38(4): 395-407.

6. Frenz P. Desafíos en Salud Pública de la Reforma: equidad y determinantes sociales de la salud. Rev Chil Salud Publica. 2005; 2(9): 103-110.

7. Krieger N. Glosario de epidemiología social. Rev Panam Salud Publica. 2002; 11(5-6): 1020-4989.

8. Faúndez A, editora. Construyendo la Nueva Agenda Social, desde la mirada de los Determinantes Sociales de la Salud. Documento Síntesis del ciclo de diálogos Democráticos. Santiago, Chile: FLAC- 
SO-Chile, OPS, Comisión de Determinantes Sociales de la Salud de la OMS y Ministerio de Salud, Gobierno de Chile; 2006. $130 \mathrm{p}$.

9. Casas JA. Salud y Desarrollo Humano: conceptos, determinantes y tareas pendientes en América Latina y el Caribe. Ginebra: División de Salud y Desarrollo Humano OPS; 2001.

10. Lalonde M. El Concepto de 'Campo de Salud': una perspectiva Canadiense. En: Organización Panamericana de la Salud. Promoción de Salud: una antología. Publicación Científica No 557. Washington, USA: OPS; 1996. p. 3.

11. Gómez H. Social determinants of health. Salud Publ Méx. 2001; 43(4): 382-383.

12. Acheson D. Independent Inquiry into Inequalities in Health. London: Stationery Office. Books; 1998. 164 p.

13. Wilkinson RG, Pickett K. The Spirit Level, Why More Equal Societies Almost Always do Better. London: Allen Lane / Penguin Press; 2009. 330 p.

14. Ziglio E, Barbosa R, Charpak Y, Turneret $\mathrm{S}$ editores. Health systems confront poverty. Copenhague: Oficina Regional de la OMS para Europa; 2003. 176 p.

15. Organización Mundial de la Salud. Informe sobre la Salud Mundial 2001. Salud mental: nuevos conocimientos y esperanzas. Ginebra: OMS; 2001.

16. Organización Mundial de la Salud. WHO global strategy on diet, physical activity and health: European regional consultation meeting report. Copenhague: OMS; 2003. 28 p.

17. Geyer S, Hemstrom O, Peter R, Vagero D. Education, income, and occupational class cannot be used interchangeably in social epidemiology. Empirical evidence against a common practice. J Epidemiol Community Health. 2006; 60(9): 804810.

18. Lahelma E, Martikainen P, Laaksonen
M, Aittomaki A. Pathways between socioeconomic determinants of health. J Epidemiol Community Health. 2004; 58: 327-332.

19. Mackenbach JP, Bakker MJ, Sihto M, Diderichsen F. Strategies to reduce socioeconomic inequalities in health. En: Mackenbach J, Bakker M, eds. Reducing Inequalities in Health: A European Perspective. London, England: Routledge; 2002: 25-50.

20. Naess O, Claussen B, Thelle DS, Davey Smith G. Four indicators of socioeconomic position: relative ranking across causes of death. Scand J Public Health. 2005; 33(3): 215-221.

21. Ministerio de Salud. Subsecretaría de Salud Pública. División de Prevención y Control de Enfermedades. Departamento de Ciclo Vital. Situación Actual de la Salud del Hombre en Chile. Santiago de Chile. [Internet] Santiago, Chile; MINSAL; 2011 [citado el 13 de diciembre de 2013]. 96 p. Disponible en: http://web. minsal.cl

22. Cabieses B, Zitko P, Pinedo R, Albor C, Espinoza MA. How to measure social position in health research? A review of international literature. Pan Journal of Public Health. 2001; 29(6): 457-68.

23. Smith D, Ebrahim S. Mendelian randomization: can genetic epidemiology contribute to understanding environmental determinants of disease? Int J Epidemiol. 2003; 32(1): 1-22.

24. Walker SN, Kerr MJ, Pender NJ, Sechrist KR. A Spanish language version of the health-promoting lifestyle profile. Nurs Res. 1990; 39(6): 268-273.

25. Pender N, Walker S, Sechrist K, Frank M. Predicting Health-Promoting Lifestyles in the Workplace. Nurs Res. 1990; 39(6): 326-332.

26. Cid P, Merino J, Stiepovich J. Factores Biológicos y Psicosociales predictores 
del estilo de vida promotor de salud. Rev Med Chil. 2006; 134(12): 1491-1499.

27. Chávez A, Vidal D, Merino J.M. Determinantes Sociales de la Salud y Conductas Vinculadas a Salud. Comuna de Coronel, Región del Bío-Bío, Chile. Cuad. médsoc. $2010 ; 50(1)$ : 39-48.

28. Finkelstein D, Mukatira S, Mehta P, Obe- nauer J, Su X, Webster R, Naeve C. Persistent host markers in pandemic and $\mathrm{H} 5 \mathrm{~N} 1$ influenza viruses. J Virol. 2007; 81(19): 10292-10299.

29. Gamarra M, Rivera H, Alcalde M, Cabellos D. Estilo de vida, autoestima y apoyo social en estudiantes de enfermería. UCV - Scientia. 2010; 2(2): 73-81. 